\title{
A Review of Amplify and Forward Bidirectional Relaying with Channel Estimation Error and Co- Channel Interference
}

\author{
Shilpa and Arvinder Pal Singh Kalsi* \\ School of Electronics and Electrical Engineering, Lovely Professional University, Phagwara - 144402, Jalandhar, \\ Punjab, India; shilpapathania9@gmail.com, arvinder.17120@lpu.co.in
}

\begin{abstract}
Objectives: To study the review of Amplify and Forward bidirectional relaying with channel estimation error and cochannel interference. Methods/Statistical analysis: This research approach is based on detailed study and analysis of two way relaying network with Amplify and Forward technique. Amplify and forward technique is preferred over decode and forward technique because it is simpler than decode and forward technique as it does not include the complex procedure of encoding and decoding process. it also includes the performance of two way relaying network or we can say bidirectional network with relay selection scheme and all relay participating case under the effect of Co-Channel Interference (CCI) and Channel Estimation Error (CEE) .Comparison between one way relaying and two way relaying is also included in this paper. Findings: Wireless communication suffers from various problems like CCI and CEE. Previous research work on bidirectional relaying networks doesn't include the effect of CC1 and CEE, but in practical, these errors exists so it is important to study the effect of these problems in our wireless communication networks. This research includes the complete survey of amplify and forward technique with one way relaying channel and two way relaying channel. It is also shown that performance of relay selection case is not always better than all relay participating case for two way relaying network. Cooperative communication is an efficient technique to deal with these types of problems by offering significant multiplexing and diversity gains without increasing power and bandwidth. Two way relaying helps to improve the overall system performance in wireless networks. Cooperative communications also deals with the various problems like fading and shadowing with the help of two way relaying. It has been proved that two way relaying is spectrally efficient technique which provides a way to surmount half duplexing loss in one way relay channel. Application/Improvements: Wireless Communication has gained a lot of interest nowadays. Our future generation requires higher data rates and a more suitable transmission with a adequate quality of service. Two Way Relaying techniques is an efficient technique to fulfill these demands with less interference.
\end{abstract}

Keywords: Amplify and Forward, Bidirectional Relaying. Co-Channel Interference, Channel Estimation Error, Cooperative Communication

\section{Introduction}

Wireless communication system has gained a lot of interest over the last years. The demand for higher data rate is increasing day by day for supporting various services that depends on internet like online games, etc $\stackrel{1}{\text {. Cooperative }}$ relaying is a diversity achieving technique which provides a reliable communication, high rate of production and large coverage in wireless network. Transmitting data over a wireless communication system suffers from various problems like fading, path loss and shadowing. These problems are compensated by various ways like increasing bandwidth or by increasing power. Or another way to compensate with these problems is to use another efficient approach known cooperative communication ${ }^{2}$.

The main objective of cooperative communication is to make non-cooperative and independent users to share their limited resources. Cooperation may be implicit 
cooperation and explicit cooperation. In implicit cooperation, pre-established framework is not required whereas explicit cooperation, it requires advanced cooperative protocols to be pre-established. Cooperation is also extendable to relaying methods which is used to extend the coverage range in wireless communication system. Cooperative communication proved to be advantageous technique, cooperation referred to as sharing of resources as well as decoding and encoding capability of the network users ${ }^{3}$.

Relaying modes are basically of two type half duplexing and full duplexing mode. Half duplexing mode takes more time than full duplexing mode for sharing same information. Generally we prefer half duplexing mode because it is less spectral efficient. Two Way Relaying (TWR) is proposed to surmount the spectral loss which occurred in half duplexing mode ${ }^{3}$.

Two Way Relaying has gained lot of interest nowadays because it provides better results and also helps to overcome the limitation that we basically faces in half duplexing. Two way relaying completes its process in two parts: first part is receive part and second part is transmit part. Multiple Input Multiple Output (MIMO) system improves diversity gain and also helps to improve capacity $^{4}$. As compare to one way relay, bidirectional relaying or we can say TWR is spectrally efficient technique for the communication among 2 users at the same time (Figure 1).

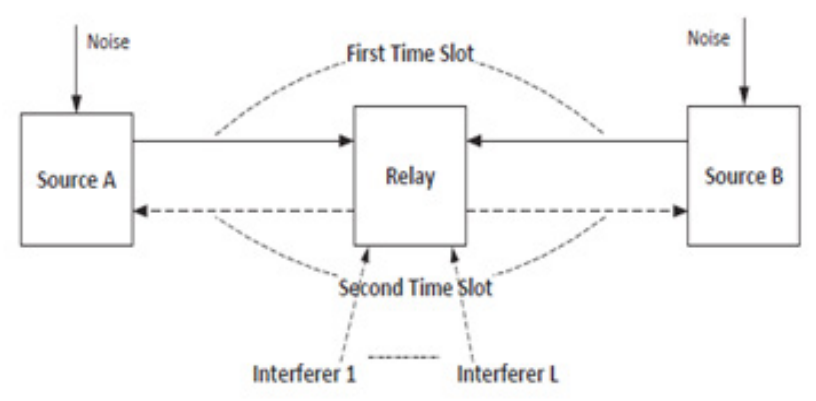

Figure 1. Bidirectional relaying system with $\mathrm{CCI}^{2}$.

Main problems from which our wireless communication system suffers are CCI and CEE. In most researches these effects are ignored and in most results, there is assumption that in second time slot two sources remove their own signals fortuitously due to assumption of perfect CCI. But in practice, these effects exist due to feedback delay and Channel Estimation Error i.e. CCI must be cal- culated therefore channel estimation error exists ${ }^{4}$. These problems degrade the system performance.

\section{Cooperative Communication}

Cooperative wireless communication is related to wireless networks where the users or wireless agents may increase their Quality of Service (QoS) with the cooperation ${ }^{5}$. Each wireless agent in a cooperative communication system transmits data and also they behave as cooperating assistant for the other wireless agent in the network ${ }^{5}$.

There is interesting tradeoffs in between transmit power and code rates. For power case, we need more power because in cooperation mode each user is transmitting for both sources, but on the other side baseline transmission power is reduced due to diversity. Therefore for power constraint tradeoffs, we expect for the net transmission power reduction, For second case code rate , similar question may arise, in cooperative communication each sends its own bits as well as some other bits of his/her partner. So, some may think that it will cause in the loss of data rates. Spectral efficiency of each user improves because of the cooperation the data rates of the channel can be increased. With the increasing number of users, demands for data rate are also increasing. The main issue which comes in cooperative communication is path from which data signal passes. For reducing this problem, the diversity technique is preferred in wireless communication system. Diversity is a phenomenon in which the signal which is transmitted travels through various paths which are independent and the possibility that all communication channel is in fade mode is negligible ${ }^{6}$. There are three basic techniques which provide diversity in wireless communication system are frequency, time and space diversity. Since last decades, wireless communication systems have been grown fortuitously. Need for higher data is increasing for fulfilling the users requirements in order to support their internet requirements like online chatting, video conferencing, online gaming etc and also to support other interactive multimedia applications. But the main problems that communication system faces are path loss, shadowing, and fading. To deal with these problems, MIMO systems are used. In MIMO systems, transmitters and receivers are accoutered with several antennas offers a new degree of freedom in spatial domain to wireless communication system. Now MIMO has become a part of various wireless communication 
standards for e.g. LTE. However use of MIMO systems in nodes having small size proved to be great challenge. To address this type of challenge, cooperative communication is used for the implementation of the concept of MIMO in a distributed manner. The concept tells that transmitting users will share each other antenna for giving virtual MIMO concept ${ }^{\mathrm{T}}$.

However, by using multiple antennas which are colocated, it will cause degradation in the QOS due to co-relation among them. Also because of the use of hardware, cost and size limitations, these small handheld devices might not be able to support multiple antennas ${ }^{8}$. These drawbacks are overcome by an approach known as cooperative communication. This type of approach is called virtual MIMO. Virtual MIMO allows single antenna to gather all the benefits of MIMO system.

With the proper utilization of the broadcasting nature, we can improve transmission performance such as throughput and reliability. With the cooperating broadcasting behavior, performance of the wireless system can be increased. Cooperative relaying is the efficient technique to deal with the fading, noise hence it enhances the system coverage and capacity.

\subsection{Basic Relaying Protocols}

In cooperative wireless communication, transmitting node transmits its own information as well as relay information on the part of each other. Process of relaying information to destination is called protocol. There are so many protocols which have already discovered, some of the relaying protocols are:

\section{A. Amplify and Forward}

Amplify and forward protocol was discovered by the Lane man et al. Here, source sends the information signal to relay node and relay amplifies that signal and forward that amplified signal to the destination.

\section{B. Decode and Forward}

It was discovered by Thomas M. cover and Abbas EI Gamal and later explored by many other scientists. In this type of relaying, user sends the data to relay and relay performs some type of decoding and checks whether the signal received is correct or wrong if it is correct then it forwards to the destination otherwise doesn't forward the signal.

\section{Compress and Forward}

In Compress and Forward type of relaying protocol, decoding of message is done at the transmitter side and a relay performs compression and sends the compressed form of signal to the destination and this technique helps in getting the benefits of the diversity technique.

\section{Literature Survey}

In practice, channel state must be estimated and therefore Channel Estimation Error exists. So, it is important to study the analysis of AF Two Way Relaying Network (TWRN) with CEE existence so that we will get the accurate and correct results. Moreover, techniques which help to remove these problems and help to enhance the system performance are of great interest. This section provides a literature survey in the AF ANC based TWRN.

TWRN has gained a precious position in wireless communication because of its benefit of surmount half duplexing loss in one way relay channel?.

There are various researches which have been already done on two way relay network with different schemes for both AF and DF network. Network Coding for TWRN was studied in ${ }^{10}$. Trade-off in Diversity multiplexing for TWRN is considered in ${ }^{11}$. The optimal distributed beam-forming structure is considered in. Asymptotic capacity analysis with multiple antennas for Two Way Relaying Network was studied in ${ }^{11}$. Cognitive spectrum sharing protocols in TWRN was discovered in ${ }^{12}$. Two Way Relaying Network which consists of several relays, there we use relay selection for multiuser diversity and this helps in improving the performance of the system. This type of RS scheme has been studied in the AF TWRN $\underline{13}$. The influence of imperfect CSI on the AF-TWRN performance was studied in ${ }^{14}$. TWRN using MIMO was studied in ${ }^{15}$. Multiple TWRN with other different signaling scheme was investigated in ${ }^{16}$. In the previous researches, the CCI effect on the TWRN is not considered. Moreover, TWRN is capable of operating in a system or surroundings which have more CCI effect operate in a rich Co-Channel interference Environment which is supposed to be an important egress in wireless communication. Therefore, we should take CCI effect in the design and in the analysis of a TWRN .Authors have recently investigated the TWRN with Amplify and Forward technique performance in the presence of CCI. Outage Probability (OP) of the TWRN with Amplify and Forward technique assumed that the relays affects from an Additive White Gaussian Noise (AWGN) and source nodes suffers from Co-Channel Interference ${ }^{\frac{17}{7}}$. Authors 
also assumed that there is no noise at the sources ${ }^{18}$. Performance of the OP for a TWRN with Amplify and Forward technique in which sources are affected by only noise and have interference limited environment ${ }^{19}$. By considering same system structure, AF TWRN performance in a Nakagami-m fading channel was investigated $\mathrm{in}^{20}$. We also observed the Co-Channel Interference effect on the AF-TWRN was studied in which nodes which are present in the wireless network are affected by both CCI as well as noise. Moreover, observations which we have concluded are totally based on the thing that the author has taken outage event as 2 different events and the whole system is not considered as one unit.

We have observed the amplifying factors are connoted to only channel gains of the link. Also, the channel gains of the assumed links are not taken. Moreover, for the AF-TWRN, most of the results assumed that two sources are capable of removing their own signal in the second time slot. Moreover, this type accuracy in self interference cancellation is based on the accuracy of Co-Channel Interference cancellation. But in reality, it is very difficult to get such an accurate Co-Channel Interference because of presence of the Channel Estimation Error and delayed feedback; it results in the decadence of performance of the system. The AF TWRN with CCI and imperfect CSI has not been reported yet in the previous research. So, nowadays researchers are focusing their research on the performance of system with CCI and imperfect CSI for enhancing system performance and for getting accurate results.

\section{Two Way Relaying (TWR)}

Cooperative Relaying is a assuring diversity achieving process, provides a higher throughput, a reliable communication and also helps in improving capacity for a wireless communication networks in a variety of applications. Two Way Relaying Network is a spectrally efficiently protocol which helps to remove the Half Duplexing Loss in One Way Relaying channel. Furthermore, by incorporating the MIMO helps to improve the diversity and spectral efficiency. The general two hop relaying protocols loss suffers from spectral efficiency loss which is overcome by TWRN. Two Way Relaying Network occupies 2 channel uses by establishing bidirectional transmission link in between communication nodes which helps to abate the loss caused by conventional two hop Relaying protocols.
In Two Way Relaying Network two sources sends information to the relay at the same time in the first time slot and in the second time slot, relay performs broadcasting of the common information to the both sources. When several relays are present, there relay selection scheme proved to be more beneficial and efficient in Two Way Relaying similar to One Way Relaying. But relay selection scheme in Two Way Relaying network is not always good when channel estimation error and co-channel interference exists ${ }^{2}$. Sometimes, we prefer all relay participating case which shows better results than relay selection scheme. Such type of observation has already been verified in $^{2}$. Figure 2 shows all relay participating case have less BER than relay selection case. Two Way Relaying network is alluring because of its cost effectiveness and higher performance. TWR improves spectral efficiency and provides significant throughput gain.

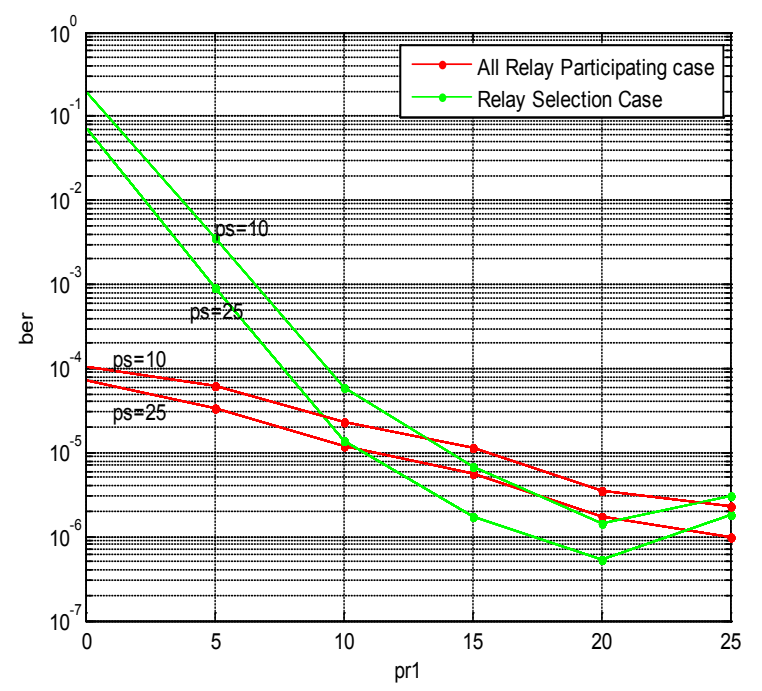

Figure 2. Comparison graph of BER vs. total relay power (pr1) with different source power (ps) for bidirectional relaying with Amplify and Forward technique under CCI and CSI effect.

\section{Conclusion}

Relay networks have acquired a very special attention nowadays. Relay networks provides a benefit for increasing spectral efficiency and for increasing capacity. TWRN as compare to One Way Relaying is more spectral efficient. In practice, we have to estimate the CSI therefore CEE exists. So it is important to study the influence of CEE on the performance of the system in AF/DF TWRN. It is also 
Table 1. Comparison between One Way Relaying (OWR) and TWR

\begin{tabular}{|c|c|c|c|}
\hline $\begin{array}{l}\text { Sl. } \\
\text { No. }\end{array}$ & Parameter & OWR & TWR \\
\hline 1 & Capacity & Capacity loss is there & No capacity loss \\
\hline 2 & Error & Decoding error is less & Decoding error is minimum than OWR \\
\hline 3 & $\begin{array}{l}\text { SNGR (Signal To Noise } \\
\text { Gap Ratio) }\end{array}$ & $\begin{array}{l}\text { Spectral efficiency is } \\
\text { less }\end{array}$ & Here spectral efficiency is more \\
\hline 4 & Spectral Efficiency & $\begin{array}{l}\text { Spectral efficiency is } \\
\text { less }\end{array}$ & Here spectral efficiency is more \\
\hline 5 & Implementation & $\begin{array}{l}\text { Practical network } \\
\text { implementation is easy }\end{array}$ & $\begin{array}{l}\text { By doing some minor changes in conventional } \\
\text { relaying system, practical network } \\
\text { implementation becomes easier }\end{array}$ \\
\hline 6 & Synchronization & $\begin{array}{l}\text { No synchronization } \\
\text { problem }\end{array}$ & $\begin{array}{l}\text { Here two user stations have to transmit data } \\
\text { simultaneously, so how to synchronize the } \\
\text { transmission is an important problem for two } \\
\text { way relaying system. }\end{array}$ \\
\hline 7 & Sum-Rate Performance & $\begin{array}{l}\text { Sum rate performance } \\
\text { is better in one way } \\
\text { relaying }\end{array}$ & $\begin{array}{l}\text { It has considerable sum rate improvement over } \\
\text { One Way Relaying (OWR). }\end{array}$ \\
\hline 8 & $\begin{array}{l}\text { No of Antennas } \\
\text { Required }\end{array}$ & $\begin{array}{l}\text { For Multiuser OWR, } \\
\text { We Require Less } \\
\text { Antennas Than TWR }\end{array}$ & $\begin{array}{l}\text { For multiuser TWR, we require more no of } \\
\text { antennas than OWR which may limit the } \\
\text { number of users to serve in practice }\end{array}$ \\
\hline
\end{tabular}

observed that relay selection scheme is not always better than all relay participating case. Moreover, the strategies for enhancing the system performance and to deal with the channel uncertainty which results from the channel estimation error are also of practical interest.

\section{References}

1. Wang C, Chen H, Yin Q, Molisch AF. Multi-user Two-Way Relay Networks with Distributed Beam Forming, IEEE Trans. Wireless Commun. 2011 Oct; 10(10):3460-71.

2. Ikki SS, Aissa S. Performance of Two-Way Amplifyand- Forward Relaying in the Presence of Co-Channel Interference, IEEE Trans. Commun. 2012 Apr; 60(4):933-39.

3. Ahn S, Oh E, Hong D. Effect of Channel Estimation Error on BER Performance in Cooperative Transmission, IEEE Trans. Veh. Technol. 2009 May; 58(4):2083-88.

4. Sha JSN, Cai YCC, Yang W. Outage Probability of Two-Way Amplify-and-Forward Relaying System with InterferenceLimited Relay, In: Proc. Wireless Commun. Signal Process, 2011.

5. Zhang S, Huang M, Zhou W. Bound Analysis of Physical Layer Network Coding in Interference-Limited Two-Way Relaying System, In Proc. IEEE Veh. Technol. Conf. Spring, 2012.
6. Liu TC-K, Wang C, Dong X. Impact of CEE on the Performance of Amplify-and-Forward Two-Way Relaying, IEEE Trans. Veh. Technol. 2012 Mar; 61(3):1197-207.

7. Wang R, Tao M. Joint Source and Relay Pre-coding Design for MIMO Two-Way Relaying Based on MSE Criteria, IEEE Trans. Signal Process. 2012 Mar; 6(3):1352-65.

8. Tao M, Wang R. Linear Pre-coding for Multi-Pair Two-Way MIMO Relay Systems with Max-Min Fairness, IEEE Trans. Signal Process. 2012 Oct; 60(10):5361-70.

9. Ikki SS, Amin O, Uysal M. Performance Analysis of Cooperative Diversity Networks with Imperfect Channel Estimation, In: Proc. IEEE ICC, 2010, p. 1-5.

10. Li J, Li W, Fan P. Network Coding for TWR Networks Over Rayleigh Fading Channels, IEEE Trans. Veh. Technol. 2010 Nov; 59(9):4476-88.

11. Chun J, Yang HJ, Paulraj A. Two Way Relay Channel with Asymptotic Capacity of the Separated MIMO, IEEE Trans. Inf. Theory. 2011 Nov; 57(11):7542-54.

12. Oh SAE, Hong D. CEE Error Effect on BER Performance in Cooperative Transmission, IEEE Trans. Veh. Technol. 2009 May; 58(4):2083-88.

13. Yoo T, Goldsmith A, Power and Capacity Allocation for Fading MIMO Channels with Channel Estimation Error, IEEE Trans. Inf. Theory. 2006 May; 52(5):2203-14.

14. Lei X, Fan P. Analysis of Outage Probability and Allocating Power for Two Way Relay Networks with Outdated Channel 
State Information and User Selection, IEEE Commun. Lett. 2012 May; 16(5):638-41.

15. Cui Q, Jantti R, Zhou M, Tao X. Two Way Relay Channel with Energy-Efficient Relay Selection and Analog Network Coding, IEEE Commun. Lett. 2012 Jun; 16(6):816-19.

16. Ji X, Zheng B, Cai Y, Zou L. On the Study of Half-Duplex Asymmetric Two-Way Relay Transmission using an Amplify-and-Forward Relay, IEEE Trans. Veh. Technol. 2012 May; 61(4):1649-63.

17. Boris Rankov, Armin Wittneben. Spectral Efficient Protocols for Half-Duplex Fading Relay Channels, IEEE J. Sel. Areas Commun. 2007 Feb; 25(2):379-89.

18. Chen M, Yener A. Multiuser Two-Way Relaying: Detection and Interference Management Strategies, IEEE Trans. Wireless Commun. 2009 Aug; 8(8):4296-305.
19. Liang X, Jin S, Wang W, Gao X, Wong K-K. Outage Probability of Amplify-and-Forward Two-Way Relay Interference-Limited Systems, IEEE Trans. Veh. Technol. 2012 Sep; 61(7):3038-49.

20. Costa D, Ding H, Yacoub MD, Ge J. TWR in InterferenceLimited AF Cooperative Networks over Nakagami-m Fading, IEEE Trans. Veh. Technol. 2012 Oct; 61(8):376671.

21. Jingon Joung, Sayed AH. Multiuser Two-Way AF Relay Processing and Power Control Methods for Beam Forming Systems, IEEE Trans. Signal Process. 2010 Mar; 58(3):1833-46. 